\title{
Normalized Coprime Factorizations in Continuous and Discrete Time - a Joint State-Space Approach
}

\author{
J. W. Hoffmann \\ Fachbereich Mathematik \\ Universität Kaiserslautern \\ Kaiserslautern, Germany
}

April 10, 1995

\begin{abstract}
Based on state-space formulas for coprime factorizations over $R H_{+}^{\infty}$ and an algebraic characterization of $J$-inner functions, normalized doubly-coprime factorizations for different classes of continuous- and discrete-time transfer functions are derived by using a single general construction method. The parametrization of the factors is in terms of the stabilizing solutions of general degenerate continuous- respectively discrete-time Riccati equations, which are obtained by examining state-space representations of $J$-normalized factor matrices.
\end{abstract}




\section{Introduction}

In this paper we study coprime factorizations of rational matrix-valued functions and their representations in state-space form. In the scalar case, every rational function $g$ with coefficients in $\mathbb{R}$ can be represented as the quotient of two coprime polynomials $p$ and $q$; a characterization of coprimeness of $p$ and $q$ is available by checking the solvability of the associated Bezout equation. For rational matrix-valued functions, factorizations are in terms of polynomial matrices, and coprimeness is again characterized via associated Bezout equations, which are now polynomial matrix equations. These polynomial coprime factorizations played an extremely important role in the development of algebraic system theory, and in particular in realization theory. Here we are interested in factorizations of rational functions over a different ring, namely over the ring $H_{+}^{\infty}$ of bounded analytic functions of the half plane respectively the unit disk.

The link between factorization theory and control problems is made via the Youla-Kucera parametrization (see e.g. Kucera (1979), Youla, Bongiorno and Jabr (1976)) which parametrizes the set of all stabilizing controllers in terms of doubly-coprime factorizations of the system matrix. This indicates why it is desirable to derive state-space formulas for coprime factorizations; those formulas first appeared in Khargonekar and Sontag (1982) and Nett, Jacobson and Balas (1984). Recently, McFarlane and Glover (1989) have pointed out that coprime factorizations which additionally satisfy certain normalization conditions are especially attractive from a computational point of view in robust control problems. Solutions to $H^{\infty}$-problems are typically iterative in nature (compare Francis (1987)). Obviously, for large systems this leads to high computational requirements; in every iteration step a set of high-dimensional Riccati equations has to be solved. However, if the nominal plant description is chosen so that the factors of the coprime factorization are normalized, it is shown by McFarlane and Glover (1989) that the optimally robust controller can be obtained without iteration, and an explicit formula for the maximum stability margin is derived.

Normalized coprime factorizations have also been used in other contexts such as in parametrization problems of linear systems (see Ober and McFarlane (1989)) or in the theory of Hankel operators (cf. e.g. Fuhrmann (1994)). The normalization conditions are adapted for different function classes depending on the special structure that the examined functions display.

In the literature, there have also been presented state-space formulas for these normalized factorizations; however, most of this work is concentrated on the case of continuous-time functions, i.e. it deals with factorizations with respect to the imaginary axis. We refer to Fuhrmann and Ober (1993), which provides a good overview of the existing literature. Moreover, in this paper there is derived a complete characterization of normalized coprime factorizations which lead to minimal McMillan degree doubly-coprime factorizations.

The literature concerning discrete-time normalized coprime factorizations, i.e. factorizations with respect to the unit disk, is by far not as rich. References known to this author containing state-space formulas for these factorizations are Meyer (1990), Chu (1988) and Bongers and Heuberger (1990). However, the first two of these papers only examine normalized factorizations for strictly proper antistable and minimal functions and additionally assume that the denominator matrices in the factorization have no pole at the origin. In the third reference, the authors treat the case of general minimal systems. 
In this paper we derive state-space formulas for normalized doubly-coprime factorizations for different classes of continuous- and discrete-time transfer functions. Normalization means $J$-innerness of the factor matrices; thus, by giving a characterization of $J$-inner functions in state-space terms and connecting these results with the formulas for general coprime factorizations over $H_{+}^{\infty}$, we obtain a parametrization of all normalized doubly-coprime factorizations in terms of the stabilizing solutions of general degenerate continuous- and discrete-time Riccati equations.

We proceed as follows. Section 2 contains some preliminaries concerning general factorizations over $H_{+}^{\infty}$ and their state-space representations. Moreover, normalized factorizations are introduced, and existence and uniqueness questions are treated using polynomial methods. In Section 3, we derive state-space characterizations for $J$-inner and $J$-coinner functions of either the half plane or the unit disk. The main result of this paper is contained in Section 4. By connecting the results of the previous sections, we obtain a link between state-space representations of $J$-normalized factorizations and degenerate Riccati

equations. This key step in the sequel immediately leads to the desired parametrization of normalized coprime factorizations. In Section 5, we finally come up with some conclusions.

\section{Preliminaries: Factorizations over $H_{+}^{\infty}$}

We start by collecting those results on factorizations of proper rational transfer functions over $R H_{+}^{\infty}$, the set of asymptotically stable proper rational matrix-valued functions, which are of relevance for the remaining paper; observe that we do not introduce different notations for $R H_{+}^{\infty}$-functions of the open left half plane $\mathbb{C}_{-}$or of the open unit disk $\mathbb{D}$. Let $L_{n}^{p, m}$ denote the class of minimal systems $(A, B, C, D) \in \mathbb{R}^{n \times n} \times \mathbb{R}^{n \times m} \times \mathbb{R}^{p \times n} \times \mathbb{R}^{p \times m}$, and let $G(s):=C(s I-A)^{-1} B+D$ be the associated transfer functions. Later in this section, it will be necessary to distinguish between minimal systems with continuous respectively discrete time axis; we denote the latter class by $D L_{n}^{p, m}$. Moreover, we introduce two subsets of $L_{n}^{p, m}\left(D L_{n}^{p, m}\right)$, namely the set $C_{n}^{p, m}\left(D_{n}^{p, m}\right)$ of continuous-time (discretetime) asymptotically stable systems respectively $A_{n}^{p, m}\left(D A_{n}^{p, m}\right)$, the class of continuoustime (discrete-time) asymptotically antistable systems. Finally, we consider two subclasses of $C_{n}^{p, m}\left(D_{n}^{p, m}\right)$; first, let $B_{n}^{p, m}\left(D B_{n}^{p, m}\right)$ denote the set of continuous-time (discrete-time) bounded real systems, which are characterized by the extra condition

$$
\begin{aligned}
& I-G^{*}(i \omega) G(i \omega)>0 \text { for all } \omega \in \mathbb{R} \cup\{ \pm \infty\} \\
& \left(I-G^{T}\left(e^{-i \Theta}\right) G\left(e^{i \Theta}\right)>0 \text { for all } \Theta \in[0,2 \pi]\right) ;
\end{aligned}
$$

furthermore, $P_{n}^{m}\left(D P_{n}^{m}\right)$ denotes the class of continuous-time (discrete-time) positive real systems, i.e. those systems in $C_{n}^{p, m}\left(D_{n}^{p, m}\right)$ which satisfy

$$
\begin{aligned}
& G(i \omega)+G^{*}(i \omega)>0 \text { for all } \omega \in \mathbb{R} \cup\{ \pm \infty\} \\
& \left(G^{T}\left(e^{-i \Theta}\right)+G\left(e^{i \Theta}\right)>0 \text { for all } \Theta \in[0,2 \pi]\right) .
\end{aligned}
$$

The notation introduced above for sets of minimal state-space systems is also used for the associated transfer function classes. Next, we analyze general factorizations of proper rational functions such that the factors are asymptotically stable, i.e. we regard factorizations with respect to the half plane in continuous-time respectively with respect to 
the unit disk in discrete-time; at first, no additional normalization condition are posed. The results summarized here were developed for the analysis of continuous-time systems in Vidyasagar (1985) and Fuhrmann and Ober (1993); however, they also hold in the discrete-time case, and hence we formulate them for the general situation.

For a proper rational matrix-valued function $G$, we consider right factorizations ( $R F$ ) $G=N M^{-1}$, where $N$ and $M$ are $R H_{+}^{\infty}$-functions and $M$ is invertible with proper inverse. If $(N, M)$ are right coprime, i.e. if there exist $R H_{+}^{\infty}$-functions $\tilde{U}$ and $\tilde{V}$ such that

$$
\tilde{V} M-\tilde{U} N=I,
$$

then the factorization is called a right coprime factorization (RCF). Analogously, left factorizations $(L F) G=\tilde{M}^{-1} \tilde{N}$ are introduced, and left coprime factorizations (LCF) are characterized via solvability of the Bezout equation

$$
\tilde{M} V-\tilde{N} U=I
$$

over $R H_{+}^{\infty}$. Vidyasagar (1985) shows that a right (left) coprime factorization of $G$ is unique up to right (left) multiplication by a $R H_{+}^{\infty}$-function with inverse in $R H_{+}^{\infty}$, i.e. a unit in $R H_{+}^{\infty}$. An existence proof for coprime factorizations using polynomial methods is contained in Fuhrmann and Ober (1993). Starting from polynomial right and left coprime factorizations, they construct right (left) factorizations $G=N M^{-1}\left(G=\tilde{M}^{-1} \tilde{N}\right)$ which additionally satisfy the degree condition

$$
\delta\left(\begin{array}{c}
M \\
N
\end{array}\right)=\delta(G) \quad\left(\delta \left(\begin{array}{cc}
-\tilde{N} & \tilde{M})=\delta(-G))
\end{array}\right.\right.
$$

here $\delta(F)$ denotes the McMillan degree of a proper rational function $F$. Observe that for general factorizations relation (5) is only true with " $\geq "$-signs. In the sequel, the authors prove that McMillan degree $n$ factors of rational functions of McMillan degree $n$ are necessarily coprime.

The next step is to derive state-space formulas for all $\mathrm{RF}$ (LF) such that equation (5) holds true; we introduce the notation $\left(\begin{array}{c|c}A & B \\ \hline C & D\end{array}\right):=C(s I-A)^{-1} B+D$. By a theorem in Vidyasagar (1985), starting from a proper rational function $G$ with minimal state-space realization $\left(\begin{array}{c|c}A & B \\ \hline C & D\end{array}\right)$, all $\mathrm{RF}$ of $G$ such that $\delta\left(\begin{array}{c}M \\ N\end{array}\right)=\delta(G)$ are given by the formula

$$
\left(\begin{array}{c}
M \\
N
\end{array}\right)=\left(\begin{array}{c|c}
A-B F & B K_{1} \\
\hline-F & K_{1} \\
C-D F & D K_{1}
\end{array}\right),
$$

where $F$ is a state-feedback such that $A-B F$ is asymptotically stable, and $K_{1}$ is an invertible matrix. On the other hand, $G=\tilde{M}^{-1} \tilde{N}$ is a LF of $G$ such that $\delta\left(\begin{array}{cc}-\tilde{N} & \tilde{M}\end{array}\right)=$ $\delta(-G)$ if and only if there exists an output injection $H$ such that $A-H C$ is asymptotically stable, and an invertible matrix $\hat{K}_{1}$ such that

$$
\left(\begin{array}{cc}
-\tilde{N} & \tilde{M}
\end{array}\right)=\left(\begin{array}{c|cc}
A-H C & H D-B & -H \\
\hline \hat{K}_{1} C & -\hat{K}_{1} D & \hat{K}_{1}
\end{array}\right) .
$$


Finally, we examine special coprime factorizations; more specifically, the two $R H_{+}^{\infty}$-block matrices

$$
\left(\begin{array}{cc}
M & U \\
N & V
\end{array}\right), \quad\left(\begin{array}{cc}
\tilde{V} & -\tilde{U} \\
-\tilde{N} & \tilde{M}
\end{array}\right)
$$

with $M$ and $\tilde{M}$ having proper inverse, form a doubly-coprime factorization of the proper rational function $G$, if $G=N M^{-1}=\tilde{M}^{-1} \tilde{N}$ and

$$
\left(\begin{array}{cc}
\tilde{V} & -\tilde{U} \\
-\tilde{N} & \tilde{M}
\end{array}\right)\left(\begin{array}{cc}
M & U \\
N & V
\end{array}\right)=\left(\begin{array}{cc}
I & 0 \\
0 & I
\end{array}\right)
$$

In addition to the formulas in (6) and (7), Fuhrmann and Ober (1993) obtain the parametrization

$$
\left(\begin{array}{cc}
M & U \\
N & V
\end{array}\right)=\left(\begin{array}{c|cc}
A-B F & B K_{1} & B D_{2}+H \hat{K}_{1}^{-1} \\
\hline-F & K_{1} & D_{2} \\
C-D F & D K_{1} & \hat{K}_{1}^{-1}+D D_{2}
\end{array}\right)
$$

and

$$
\left(\begin{array}{cc}
\tilde{V} & -\tilde{U} \\
-\tilde{N} & \tilde{M}
\end{array}\right)=\left(\begin{array}{c|cc}
A-H C & H D-B & -H \\
\hline-K_{1}^{-1} F-K_{1}^{-1} D_{2} \hat{K}_{1} C & K_{1}^{-1}+K_{1}^{-1} D_{2} \hat{K}_{1} D & -K_{1}^{-1} D_{2} \hat{K}_{1} \\
\hat{K}_{1} C & -\hat{K}_{1} D & \hat{K}_{1}
\end{array}\right) \text { (9) }
$$

(with $D_{2}$ arbitrary) for all doubly-coprime factorizations of $G$ such that

$$
\delta\left(\begin{array}{cc}
M & U \\
N & V
\end{array}\right)=n=\delta\left(\begin{array}{cc}
\tilde{V} & -\tilde{U} \\
-\tilde{N} & \tilde{M}
\end{array}\right)
$$

Before we start with the analysis of coprime factorizations with an extra normalization condition, we have to point out a main difference in the analysis of continuous- and discrete-time systems; up to now the development has been completely parallel for both cases. Taking the adjoint of a continuous-time transfer function $G(s) \in L_{n}^{p, m}$ in the $L^{2}(i \mathbb{R})$-scalar product, we get the conjugate system $G^{*}(s)$ of $G(s)$, which is given by the formula

$$
G^{*}(s)=G^{*}(-\bar{s}) .
$$

In discrete-time, however, the adjoint is taken with respect to the scalar product in $L^{2}(\delta \mathbb{D})$ (where $\delta \mathbb{D}:=\{z:|z|=1\}$ ), and hence here we get

$$
G^{*}(z)=G^{*}\left(\bar{z}^{-1}\right)
$$

for the conjugate system $G^{*}(z)$ of a discrete-time transfer function $G(z) \in D L_{n}^{p, m}$; in equation (10) as well as in equation (11), the symbol "*" on the right hand side denotes the complex conjugate transpose.

Now we can proceed to formulate the normalization conditions; these conditions are adapted for different function classes depending on the special structure the examined functions display. 
Definition 2.1 Let $G$ be a proper rational function, and let $G=N M^{-1}\left(G=\tilde{M}^{-1} \tilde{N}\right)$ be a right (left) factorization. Then the factorization is called a $J$-normalized right (left) factorization, write $J-R F(J-L F)$, if

$$
\begin{aligned}
& \left(\begin{array}{ll}
M^{*} & N^{*}
\end{array}\right) J\left(\begin{array}{c}
M \\
N
\end{array}\right)=I \\
& \left(\left(\begin{array}{ll}
\tilde{M} & \tilde{N}
\end{array}\right) J\left(\begin{array}{c}
\tilde{M}^{*} \\
\tilde{N}^{*}
\end{array}\right)=I\right),
\end{aligned}
$$

where for the different function classes we have:

(i) if $G(s) \in A_{n}^{p, m}$ or $G(s) \in D A_{n}^{p, m}$, then $J=J_{S}:=\left(\begin{array}{cc}I & 0 \\ 0 & 0\end{array}\right)$;

(ii) if $G(s) \in L_{n}^{p, m}$ or $G(s) \in D L_{n}^{p, m}$, then $J=J_{L}:=\left(\begin{array}{cc}I & 0 \\ 0 & I\end{array}\right)$;

(iii) if $G(s) \in B_{n}^{p, m}$ or $G(s) \in D B_{n}^{p, m}$, then $J=J_{B}:=\left(\begin{array}{cc}I & 0 \\ 0 & -I\end{array}\right)$;

(iv) if $G(s) \in P_{n}^{m}$ or $G(s) \in D P_{n}^{m}$, then $J=J_{P}:=\left(\begin{array}{cc}0 & I \\ I & 0\end{array}\right)$.

$J_{L}, J_{B}$ and $J_{P}$ introduce an indefinite inner product on $L^{2}(i \mathbb{R}) \oplus L^{2}(i \mathbb{R})\left(L^{2}(\delta \mathbb{D}) \oplus L^{2}(\delta \mathbb{D})\right)$, whereas $J_{S}$ introduces a degenerate metric; for the theory of general inner product spaces or Krein spaces we refer to Bognar (1974). We fix the following notation for the rest of this paper: by $\mathcal{C}$ we denote any element of the set $\left\{A_{n}^{p, m}, D A_{n}^{p, m}, L_{n}^{p, m}, D L_{n}^{p, m}, B_{n}^{p, m}, D B_{n}^{p, m}\right.$, $\left.P_{n}^{m}, D P_{n}^{m}\right\}$, and by $J_{\mathcal{C}}$ we denote the associated metric (according to Definition 2.1).

Next, the question of existence and uniqueness of such normalized factorizations has to be treated. In the continuous-time case, Fuhrmann and Ober (1993) contains a complete answer to these questions; the way of proof is constructive using polynomial methods. Below, we formulate their theorem also for the discrete-time case and give a proof for this case using the so-called Moebius transform. This is a standard device used to relate functions analytic in a half plane to those analytic in the unit circle (see Hoffmann (1962)). More specifically, the transformation

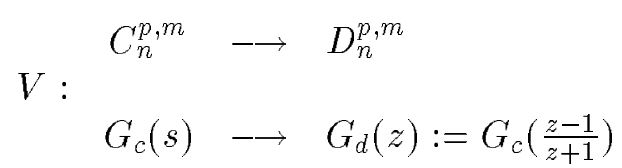

is a bijection with inverse

$$
\begin{aligned}
V^{-1}: & \longrightarrow C_{n}^{p, m} \\
G_{d}(z) & \longrightarrow G_{c}(s):=G_{d}\left(\frac{1+s}{1-s}\right) .
\end{aligned}
$$

Via this bijection there are induced bijections between $B_{n}^{p, m}$ and $D B_{n}^{p, m}$ respectively $P_{n}^{m}$ and $D P_{n}^{m}$. 
Theorem 2.1 Let $G \in \mathcal{C}$ be a proper rational function. Then there exists a $J_{\mathcal{C}}$-right (left) factorization $G=N M^{-1}\left(G=\tilde{M}^{-1} \tilde{N}\right)$. All $J_{\mathcal{C}}$-factorizations are coprime and such that $\delta\left(\begin{array}{c}M \\ N\end{array}\right)=n\left(\delta\left(\begin{array}{ll}\tilde{M} & -\tilde{N}\end{array}\right)=n\right)$; moreover, the right (left) $J_{\mathcal{C}}$-factorizations are unique up to right (left) multiplication by a constant unitary matrix.

\section{Proof (discrete-time case):}

We only prove the statement for right factorizations; a completely analogous argumentation works for left factorizations. The inverse Moebius transform maps $G(z)$ into a function $G_{c}(s):=G\left(\frac{1+s}{1-s}\right)$ of McMillan degree $n$, and $G_{c}$ belongs to the continuous-time analogue of $\mathcal{C}$. Let $G_{c}(s)=N_{c}(s) M_{c}^{-1}(s)$ be the corresponding continuous-time $J_{\mathcal{C}}$-normalized right factorization; the existence of such factorizations with $M_{c}^{-1}(s)$ proper and $\delta\left(\begin{array}{c}M_{c} \\ N_{c}\end{array}\right)=n$ has been shown in Fuhrmann and Ober (1993). Let

$$
N(z):=N_{c}\left(\frac{z-1}{z+1}\right), \quad M(z):=M_{c}\left(\frac{z-1}{z+1}\right)
$$

be the functions obtained by application of the transformation $V$. Then in view of the properties of the Moebius transform $N$ and $M$ are discrete-time asymptotically stable with $\delta\left(\begin{array}{c}M \\ N\end{array}\right)=n$ and

$$
G(z)=G_{c}\left(\frac{z-1}{z+1}\right)=N_{c}\left(\frac{z-1}{z+1}\right) M_{c}^{-1}\left(\frac{z-1}{z+1}\right)=N(z) M^{-1}(z),
$$

i.e. $(N, M)$ constitute a right coprime factorization of $G$. Furthermore,

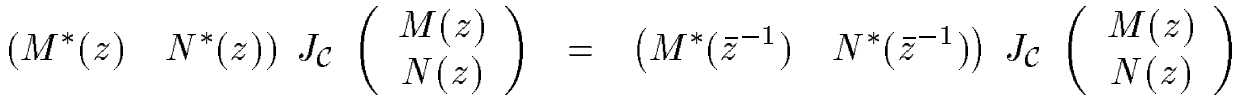

$$
\begin{aligned}
& =\left.\left(\begin{array}{lll}
M_{c}^{*}(-\bar{s}) & N_{c}^{*}(-\bar{s})
\end{array}\right) J_{\mathcal{C}}\left(\begin{array}{c}
M_{c}(s) \\
N_{c}(s)
\end{array}\right)\right|_{s=\frac{s-1}{s+1}}
\end{aligned}
$$

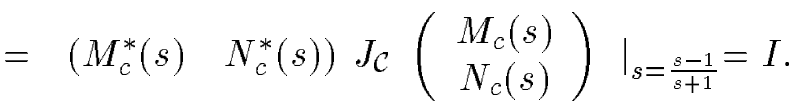

Hence $G=N M^{-1}$ really is a $J_{\mathcal{C}}-\mathrm{RCF}$. The statement concerning uniqueness is easily proved using Vidyasagar's result on the uniqueness properties of general coprime factorizations and the $J_{\mathcal{C}}$-normalization condition.

This way of proof obviously fails when $G(z)$ is an element of $D L_{n}^{p, m}$ and has a pole at $z=-1$, because then $G_{c}(s)$ is not proper. As an easy SISO example take $g(z)=\frac{1}{z+1}$; then $g_{c}(s)=g\left(\frac{1+s}{1-s}\right)=\frac{1-s}{2}$. However, one can use a different bilinear transformation $z \mapsto \frac{p z+q}{r z+t}$ with $p, q, r, t \in \mathbb{C}$ and $p t-r q \neq 0$ (see e.g. Bart, Gohberg and Kaashoek (1979)) in this case; the inverse transformation is then given by $s \mapsto \frac{-t s+q}{r s-p}$. If one chooses the parameters such that the denominator has its singularity on the unit disk, then again $\mathbb{C}_{-}$is mapped onto $\mathbb{D}$, and if we additionally select the singularity such that it does not coincide with a pole of $G$, then the above proof can be performed analogously using the modified Moebius transformation. 


\section{Characterization of $J$-inner functions}

Taking a closer look at Definition 2.1 it becomes apparent that the normalization conditions for the special right (left) factorizations mean nothing else than postulating that the matrices composed by the factors are $J_{\mathcal{C}}$-inner $\left(J_{\mathcal{C}}\right.$-coinner $)$ functions. Here we call a rational matrix-valued function $U(s) \in L_{n}^{p, m}\left(D L_{n}^{p, m}\right) J$-inner, if $U(s) \in C_{n}^{p, m}\left(D_{n}^{p, m}\right)$ and

$$
U^{*}(s) J U(s)=I
$$

we remind the reader of the different meanings of conjugation for continuous- respectively discrete-time functions. Moreover, $U(s) \in L_{n}^{p, m}\left(D L_{n}^{p, m}\right)$ is called $J$-coinner, if $U(s) \in$ $C_{n}^{p, m}\left(D_{n}^{p, m}\right)$ and

$$
U(s) J U^{*}(s)=I .
$$

State-space characterizations of inner functions $(J=I)$ have appeared at several places in the control literature; we refer e.g. to Glover (1984), where the case of square continuous-time transfer functions is treated. Here we present a generalized result for nonsquare systems and arbitrary metric $J$. The proof uses some well-known facts about the solution set of continuous-time Lyapunov equations, or, equivalently, the Sylvester map

$$
\begin{array}{rll}
S: \begin{array}{l}
\mathbb{C}^{n \times m} \\
V
\end{array} & \longrightarrow \mathbb{C}^{n \times m}
\end{array} \quad, A_{1} V-V A_{2} \quad \mathbb{C}^{n \times n}, A_{2} \in \mathbb{C}^{m \times m} ;
$$

we remind the reader that $S$ is an isomorphism if and only if $\sigma\left(A_{1}\right) \cap \sigma\left(A_{2}\right)=\emptyset$, where $\sigma\left(A_{i}\right)$ is the spectrum of $A_{i}$ (cf. e.g. Wonham (1979)).

Theorem 3.1 (continuous-time case) Let

$$
U=\left(\begin{array}{l|l}
A & B \\
\hline C & D
\end{array}\right)
$$

be a transfer function with $\sigma(A) \subseteq \mathbb{C}_{-}$, and let $J$ be a given metric. Then:

(i) (a) Assume there exists a solution $X$ of the system

$$
\begin{aligned}
A^{*} X+X A+C^{*} J C & =0, \\
D^{*} J C+B^{*} X & =0, \\
D^{*} J D & =I,
\end{aligned}
$$

then $U$ is $J$-inner.

(b) If $U$ is $J$-inner and the realization (19) is reachable, then there exists a symmetric solution $X$ of the system (CIn1)-(CIn3).

(ii) (a) Assume there exists a solution $Y$ of the system

$$
\begin{aligned}
A Y+Y A^{*}+B J B^{*} & =0, \\
B J D^{*}+Y C^{*} & =0, \\
D J D^{*} & =I,
\end{aligned}
$$

then $U$ is $J$-coinner. 
(b) If $U$ is $J$-coinner and the realization (19) is observable, then there exists a symmetric solution $Y$ of the system (CIn 4$)-(C I n 6)$.

Proof:

Starting with a realization of $U(s)$ as given in (19), we calculate

$$
U^{*}(s)=U^{*}(-\bar{s})=\left(\begin{array}{l|l}
-A^{*} & C^{*} \\
\hline-B^{*} & D^{*}
\end{array}\right)
$$

and

$$
U^{*}(s) J U(s)=\left(\begin{array}{cc|c}
A & 0 & B \\
C^{*} J C & -A^{*} & C^{*} J D \\
\hline D^{*} J C & -B^{*} & D^{*} J D
\end{array}\right) .
$$

Define the state-space transformation

$$
T_{Q}:=\left(\begin{array}{cc}
I & 0 \\
-Q & I
\end{array}\right)
$$

where $Q$ is an arbitrary (square) matrix of appropriate size. Then in new coordinates we get

$$
T_{Q}^{-1}\left(\begin{array}{cc}
A & 0 \\
C^{*} J C & -A^{*}
\end{array}\right) T_{Q}=\left(\begin{array}{cc}
A & 0 \\
Q A+A^{*} Q+C^{*} J C & -A^{*}
\end{array}\right) .
$$

Moreover, one calculates

$$
T_{Q}^{-1}\left(\begin{array}{c}
B \\
C^{*} J D
\end{array}\right)=\left(\begin{array}{c}
B \\
Q B+C^{*} J D
\end{array}\right)
$$

and

$$
\left(\begin{array}{ll}
D^{*} J C & -B^{*}
\end{array}\right) T_{Q}=\left(\begin{array}{ll}
D^{*} J C+B^{*} Q & -B^{*}
\end{array}\right) .
$$

This results in the following representation for $U^{*} J U$ in new coordinates:

$$
U^{*}(s) J U(s)=\left(\begin{array}{cc|c}
A & 0 & B \\
Q A+A^{*} Q+C^{*} J C & -A^{*} & Q B+C^{*} J D \\
\hline B^{*} Q+D^{*} J C & -B^{*} & D^{*} J D
\end{array}\right) .
$$

(i)(a): Now assume that equations (CIn1)-(CIn3) hold true; observe that $X$, the solution to this set of equations, is symmetric, since $X^{*}$ also solves the Lyapunov equation (CIn1), and the solution to this equation is uniquely determined because the Sylvester map is isomorphic for asymptotically stable $A$. By applying the state-space transformation $T_{X}$, equation (22) immediately reduces to

$$
U^{*}(s) J U(s)=\left(\begin{array}{cc|c}
A & 0 & B \\
0 & -A^{*} & 0 \\
\hline 0 & -B^{*} & I
\end{array}\right)=I .
$$

(i)(b): To show the converse, assume that $U^{*}(s) J U(s)=I$ holds true and that the realization (19) is reachable; furthermore, let $X$ denote the uniquely determined (symmetric) 
solution of the Lyapunov equation (CIn1); the existence of such an $X$ is obvious from the properties of the Sylvester map. By (22), application of the coordinate transformation $T_{X}$ to the representation (21) yields

$$
I=U^{*}(s) J U(s)=\left(\begin{array}{cc|c}
A & 0 & B \\
0 & -A^{*} & X B+C^{*} J D \\
\hline B^{*} X+D^{*} J C & -B^{*} & D^{*} J D
\end{array}\right) .
$$

This immediately implies for the feedthrough term that $D^{*} J D=I$, i.e. (CIn3) holds true. Hence it only remains to show that also equation (CIn2) is satisfied. Observe that for the strictly proper part of $U^{*} J U$ we have

$$
\left(\begin{array}{cc|c}
A & 0 & B \\
0 & -A^{*} & X B+C^{*} J D \\
\hline B^{*} X+D^{*} J C & -B^{*} & 0
\end{array}\right)=F(s)+F^{*}(s),
$$

where

$$
F(s):=\left(\begin{array}{c|c}
A & B \\
\hline B^{*} X+D^{*} J C & 0
\end{array}\right) .
$$

Thus, (22) yields that

$$
F(s)=-F^{*}(s) \text {. }
$$

Now the asymptotic stability of $A$ implies that $F(s)$ is asymptotically stable, too; hence $F^{*}(s)$ is asymptotically antistable, which in view of equation (24) means that $F(s)$ has to be constant, i.e. equal to zero because of the strict properness of $F(s)$. Looking at the coefficients in the power series expansion of $F(s)$ indicates that

$$
\left(B^{*} X+D^{*} J C\right)\left(\begin{array}{c}
B \\
A B \\
A^{2} B \\
\vdots
\end{array}\right)=0
$$

which in view of the reachability of the pair $(A, B)$ gives $B^{*} X+D^{*} J C=0$, i.e. equation (CIn2) is true.

(ii): Analogous.

Below, we state the corresponding result for discrete-time $J$-normalized functions.

Theorem 3.2 (discrete-time case) Let

$$
U=\left(\begin{array}{l|l}
A & B \\
\hline C & D
\end{array}\right)
$$

be a transfer function with $\sigma(A) \subseteq \mathbb{D}$, and let $J$ be a given metric. Then:

(i) (a) Assume there exists a solution $X$ of the system

$$
\begin{aligned}
A^{*} X A+C^{*} J C & =X, \\
D^{*} J C+B^{*} X A & =0, \\
D^{*} J D+B^{*} X B & =I,
\end{aligned}
$$

then $U$ is $J$-inner. 
(b) If $U$ is $J$-inner and the realization (25) is reachable, then there exists a symmetric solution $X$ of the system (DIn1)-(DIn3).

(ii) (a) Assume there exists a solution $Y$ of the system

$$
\begin{aligned}
& A Y A^{*}+B J B^{*}=Y, \\
& B J D^{*}+A Y C^{*}=0, \\
& D J D^{*}+C Y C^{*}=I,
\end{aligned}
$$

then $U$ is $J$-coinner.

(b) If $U$ is $J$-coinner and the realization (25) is observable, then there exists a symmetric solution $Y$ of the system (DIn4)-(DIn6).

A proof for the case of inner functions $(J=I)$ can be found in Fuhrmann and Hoffmann (1994). In view of the formula for conjugation in the discrete-time case (compare (11)), $U^{*}(z)$ will be proper rational if and only if $U(z)$ has no pole at the origin; a simple example of an inner function with nonproper conjugate function is $U(z)=\frac{1}{z}$. So only in the special case of no pole at zero we have a state-space formula similar to (20) for the associated conjugate system at our disposal. This means that in general the proof in the discrete-time case can not be performed using state-space arguments; instead, Fuhrmann and Hoffmann (1994) use a power series approach. This method of proof easily carries over to the case of general metric $J$, if one takes into account the following lemma (and its dual version); we remind the reader that the discrete-time analogue of the Sylvester map, the so-called Stein map

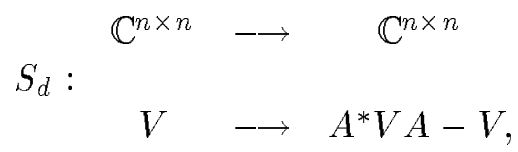

for $A \in \mathbb{C}^{n}$ with $\sigma(A) \subseteq \mathbb{D}$ is an isomorphism.

Lemma 3.1 Let $A$ with $\sigma(A) \subseteq \mathbb{D}$ be given, and let $C$ arbitrary. Then the equation

$$
X=A^{*} X A+C^{*} J C
$$

has the uniquely determined symmetric solution

$$
X=\sum_{i=1}^{\infty}\left(A^{*}\right)^{i-1} C^{*} J C A^{i-1} .
$$

\section{Proof:}

As we mentioned above, for asymptotically stable $A$ the map $X \longrightarrow X-A^{*} X A$ is an isomorphism, and hence a solution to (27) exists and is uniquely determined. Obviously, for a solution $X$ of (27) there holds

$$
X^{*}=\left(A^{*} X A+C^{*} J C\right)^{*}=A^{*} X^{*} A+C^{*} J C,
$$

which indicates that $X^{*}$ also solves (27), and because of the uniqueness of the solution we get $X=X^{*}$. Finally, we show that the solution is of the form (28). Define

$$
X_{n}:=C^{*} J C+A^{*} C^{*} J C A+\ldots+\left(A^{*}\right)^{n} C^{*} J C A^{n}
$$


for $n \geq 0$. Then

$$
X_{n}-A^{*} X_{n} A=C^{*} J C-\left(A^{*}\right)^{n+1} C^{*} J C A^{n+1}
$$

and

$$
X_{n+1}-X_{n}=\left(A^{*}\right)^{n+1} C^{*} J C A^{n+1} .
$$

Since $\sigma(A) \subseteq \mathbb{D}$, we have

$$
\|A\|^{n+1} \longrightarrow 0 \quad \text { for } n \longrightarrow \infty
$$

moreover,

$$
\left\|\left(A^{*}\right)^{n+1} C^{*} J C A^{n+1}\right\| \leq\left\|A^{*}\right\|^{n+1}\left\|C^{*} J C\right\|\|A\|^{n+1}
$$

using a submultiplicative matrix norm. It therefore follows from (31) that

$$
\left(A^{*}\right)^{n+1} C^{*} J C A^{n+1} \longrightarrow 0 \quad \text { for } n \longrightarrow \infty ;
$$

additionally, taking the spectral norm on $\mathbb{C}^{n \times n}$, we conclude that

$$
\left\|\left(A^{*}\right)^{n+1} C^{*} J C A^{n+1}\right\| \leq \kappa|\lambda|^{2(n+1)},
$$

where $\lambda$ with $|\lambda|<1$ is the largest eigenvalue of $A$ and $\kappa$ is constant. Hence, the convergence of the geometric series together with (30) implies that the limit

$$
X:=\lim _{n \mapsto \infty} X_{n}=\sum_{i=1}^{\infty}\left(A^{*}\right)^{i-1} C^{*} J C A^{i-1}
$$

exists; finally, (29) shows that the series $X$ is a solution of equation (27).

\section{Main result}

In Section 2 we have presented state-space formulas for coprime factorizations without an additional normalization condition, and Section 3 contains a state-space characterization of $J_{\mathcal{C}}$-inner respectively $J_{\mathcal{C}}$-coinner functions. As we already pointed out earlier, the various normalization conditions in Definition 2.1 express the fact that the factor matrices have to be $J_{\mathcal{C}}$-inner functions. Hence by connecting the results in Section 2 and 3, we obtain the desired state-space formulas for the normalized coprime factorizations.

The first step is to investigate how $J$-innerness relates to the solvability of a certain degenerate Riccati equation. We start with right factorizations in continuous-time; although the subsequent lemmata are of rather technical nature, they constitute the key tools for the derivation of the main result later in this section.

Lemma 4.1 (continuous-time case) Let

$$
\left(\begin{array}{c}
M \\
N
\end{array}\right)=\left(\begin{array}{c|c}
A-B F & B K_{1} \\
\hline-F & K_{1} \\
C-D F & D K_{1}
\end{array}\right)
$$


be a realization of the asymptotically stable function $\left(\begin{array}{c}M \\ N\end{array}\right)$, and let $M^{-1}$ be proper rational; moreover, let $J$ be a given metric and define

$$
W:=\left[\left(\begin{array}{l}
I \\
D
\end{array}\right)^{*} J\left(\begin{array}{l}
I \\
D
\end{array}\right)\right] .
$$

(i) Assume that the realization (32) is reachable, $\sigma(A-B F) \subseteq \mathbb{C}_{-}$and $\left(\begin{array}{l}M \\ N\end{array}\right)$ is $J_{-}$ inner. Then there exists a solution $X$ to the system of equations

$$
\begin{aligned}
(A-B F)^{*} X+X(A-B F)+\left(\begin{array}{c}
-F \\
C-D F
\end{array}\right)^{*} J\left(\begin{array}{c}
-F \\
C-D F
\end{array}\right) & =0 \\
\left(\begin{array}{c}
K_{1} \\
D K_{1}
\end{array}\right)^{*} J\left(\begin{array}{c}
-F \\
C-D F
\end{array}\right)+\left(B K_{1}\right)^{*} X & =0 \\
\left(\begin{array}{c}
K_{1} \\
D K_{1}
\end{array}\right)^{*} J\left(\begin{array}{c}
K_{1} \\
D K_{1}
\end{array}\right) & =I .
\end{aligned}
$$

Furthermore, $W$ is invertible, and there holds

$$
K_{1}=W^{-\frac{1}{2}} D_{1}
$$

where $D_{1}^{*} D_{1}=I$,

$$
F=W^{-1}\left[\left(\begin{array}{l}
I \\
D
\end{array}\right)^{*} J\left(\begin{array}{c}
0 \\
C
\end{array}\right)+B^{*} X\right]=: W^{-1} L
$$

and $X$ is a stabilizing solution to the Riccati equation

$$
\begin{aligned}
0=A^{*} X+X A+\left(\begin{array}{c}
0 \\
C
\end{array}\right)^{*} J\left(\begin{array}{c}
0 \\
C
\end{array}\right)-\left[X B+\left(\begin{array}{l}
0 \\
C
\end{array}\right)^{*} J\left(\begin{array}{c}
I \\
D
\end{array}\right)\right] \\
\cdot\left[\left(\begin{array}{c}
I \\
D
\end{array}\right)^{*} J\left(\begin{array}{c}
I \\
D
\end{array}\right)\right]^{-1}\left[\left(\begin{array}{c}
I \\
D
\end{array}\right)^{*} J\left(\begin{array}{c}
0 \\
C
\end{array}\right)+B^{*} X\right],
\end{aligned}
$$

i.e. $X$ solves (39) and

$$
A-B\left[\left(\begin{array}{l}
I \\
D
\end{array}\right)^{*} J\left(\begin{array}{l}
I \\
D
\end{array}\right)\right]^{-1}\left[\left(\begin{array}{c}
I \\
D
\end{array}\right)^{*} J\left(\begin{array}{c}
0 \\
C
\end{array}\right)+B^{*} X\right]
$$

has all eigenvalues in $\mathbb{C}_{-}$.

(ii) Conversely, assume that $W$ is invertible and that there exists a stabilizing solution $X$ to the Riccati equation (39); moreover, let the parameters $K_{1}$ and $F$ in the realization (32) be given by the formulas (37) respectively (38). Then $\left(\begin{array}{l}M \\ N\end{array}\right)$ is J-inner, and $X$ solves the system of equations (34)-(36). 


\section{Proof:}

(i): Assume that $\left(\begin{array}{c}M \\ N\end{array}\right)$ is $J$-inner, $A-B F$ is asymptotically stable and the realization (32) is reachable. Then by Theorem 3.1,(i)(b) there exists a solution $X$ of the system (CIn1)-(CIn3), which for the given realization are equations (34)-(36). Next, we obtain from (36) that

$$
I=\left(\begin{array}{c}
K_{1} \\
D K_{1}
\end{array}\right)^{*} J\left(\begin{array}{c}
K_{1} \\
D K_{1}
\end{array}\right)=K_{1}^{*}\left[\left(\begin{array}{c}
I \\
D
\end{array}\right)^{*} J\left(\begin{array}{c}
I \\
D
\end{array}\right)\right] K_{1}
$$

which immediately implies that $W$ is invertible and $K_{1}$ is of the form (37). Moreover, equation (35) reads

$$
0=\left(\begin{array}{c}
K_{1} \\
D K_{1}
\end{array}\right)^{*} J\left(\begin{array}{c}
-F \\
C-D F
\end{array}\right)+\left(B K_{1}\right)^{*} X=K_{1}^{*}\left[\left(\begin{array}{c}
I \\
D
\end{array}\right)^{*} J\left(\begin{array}{c}
-F \\
C-D F
\end{array}\right)+B^{*} X\right]
$$

which in view of the invertibility of $K_{1}$ gives

$$
\begin{aligned}
0 & =\left(\begin{array}{l}
I \\
D
\end{array}\right)^{*} J\left(\begin{array}{c}
-F \\
C-D F
\end{array}\right)+B^{*} X \\
& =\left[\left(\begin{array}{c}
I \\
D
\end{array}\right)^{*} J\left(\begin{array}{c}
-I \\
-D
\end{array}\right)\right] F+\left(\begin{array}{c}
I \\
D
\end{array}\right)^{*} J\left(\begin{array}{c}
0 \\
C
\end{array}\right)+B^{*} X
\end{aligned}
$$

or

$$
\left[\left(\begin{array}{l}
I \\
D
\end{array}\right)^{*} J\left(\begin{array}{c}
I \\
D
\end{array}\right)\right] F=\left(\begin{array}{c}
I \\
D
\end{array}\right)^{*} J\left(\begin{array}{c}
0 \\
C
\end{array}\right)+B^{*} X
$$

Using the invertibility of $W$ as established above yields formula (38) for $F$. The next step is to show that $X$ is a stabilizing solution of the Riccati equation (39). We get from (34) that

$$
\begin{aligned}
0= & (A-B F)^{*} X+X(A-B F)+\left(\begin{array}{c}
-F \\
C-D F
\end{array}\right)^{*} J\left(\begin{array}{c}
-F \\
C-D F
\end{array}\right) \\
= & A^{*} X+X(A-B F)-F^{*}\left[B^{*} X+\left(\begin{array}{c}
I \\
D
\end{array}\right)^{*} J\left(\begin{array}{c}
-F \\
C-D F
\end{array}\right)\right] \\
& +\left(\begin{array}{c}
0 \\
C
\end{array}\right)^{*} J\left(\begin{array}{c}
-F \\
C-D F
\end{array}\right),
\end{aligned}
$$

which by (41) reduces to

$$
\begin{aligned}
0 & =A^{*} X+X(A-B F)+\left(\begin{array}{l}
0 \\
C
\end{array}\right)^{*} J\left(\begin{array}{c}
-F \\
C-D F
\end{array}\right) \\
& =A^{*} X+X A+\left(\begin{array}{l}
0 \\
C
\end{array}\right)^{*} J\left(\begin{array}{l}
0 \\
C
\end{array}\right)-\left[X B+\left(\begin{array}{l}
0 \\
C
\end{array}\right)^{*} J\left(\begin{array}{l}
I \\
D
\end{array}\right)\right] F
\end{aligned}
$$

This shows that $X$ solves the Riccati equation, since we have shown above that $F$ is of the form (38). Finally, it is clear that $X$ is stabilizing, since $A-B F$ is asymptotically stable by assumption and has the form (40).

(ii): Observe that by Theorem 3.1,(i)(a) and the asymptotic stability of $A-B F$ the 
solvability of the system (34)-(36) is a sufficient condition for $\left(\begin{array}{c}M \\ N\end{array}\right)$ being $J$-inner; indeed, these equations are nothing else but the equations (CIn1)-(CIn3) in terms of the given realization $(32)$ of $\left(\begin{array}{c}M \\ N\end{array}\right)$. Hence we only have to show that equations (34)-(36) hold. So assume that $K_{1}$ and $F$ are given as in (37) respectively (38) with $X$ a stabilizing solution of (39); we remark that $K_{1}$ is necessarily invertible because $M^{-1}$ is proper rational. We start by checking equation (36); looking at (37) there holds

$$
\begin{aligned}
\left(\begin{array}{c}
K_{1} \\
D K_{1}
\end{array}\right)^{*} J\left(\begin{array}{c}
K_{1} \\
D K_{1}
\end{array}\right) & =K_{1}^{*}\left[\left(\begin{array}{c}
I \\
D
\end{array}\right)^{*} J\left(\begin{array}{c}
I \\
D
\end{array}\right)\right] K_{1} \\
& =K_{1}^{*} W K_{1}=D_{1}^{*} W^{-\frac{1}{2}} W W^{-\frac{1}{2}} D_{1}=D_{1}^{*} D_{1}=I .
\end{aligned}
$$

Next, we obtain by (38) that

$$
\begin{array}{rl}
\left(\begin{array}{c}
K_{1} \\
D K_{1}
\end{array}\right)^{*} & J\left(\begin{array}{c}
-F \\
C-D F
\end{array}\right)+\left(B K_{1}\right)^{*} X \\
& =K_{1}^{*}\left[\left(\begin{array}{c}
I \\
D
\end{array}\right)^{*} J\left(\begin{array}{c}
-F \\
C-D F
\end{array}\right)+B^{*} X\right] \\
& =K_{1}^{*}\left[\left(\begin{array}{c}
I \\
D
\end{array}\right)^{*} J\left(\begin{array}{c}
0 \\
C
\end{array}\right)-\left(\begin{array}{c}
I \\
D
\end{array}\right)^{*} J\left(\begin{array}{c}
I \\
D
\end{array}\right) F+B^{*} X\right] \\
& =K_{1}^{*}\left[L-\left(\begin{array}{c}
I \\
D
\end{array}\right)^{*} J\left(\begin{array}{c}
I \\
D
\end{array}\right) F\right]=0
\end{array}
$$

thus also (35) holds true. Multiplying (35) on the left by $\left(K_{1}^{*}\right)^{-1}$ we finally calculate

$$
\begin{aligned}
(A-B F)^{*} X+X(A-B F)+\left(\begin{array}{c}
-F \\
C-D F
\end{array}\right)^{*} J\left(\begin{array}{c}
-F \\
C-D F
\end{array}\right) \\
=A^{*} X+X(A-B F)-F^{*}\left[B^{*} X+\left(\begin{array}{c}
I \\
D
\end{array}\right)^{*} J\left(\begin{array}{c}
-F \\
C-D F
\end{array}\right)\right] \\
\quad+\left(\begin{array}{c}
0 \\
C
\end{array}\right)^{*} J\left(\begin{array}{c}
-F \\
C-D F
\end{array}\right) \\
=A^{*} X+X(A-B F)+\left(\begin{array}{l}
0 \\
C
\end{array}\right)^{*} J\left(\begin{array}{c}
-F \\
C-D F
\end{array}\right) \\
=A^{*} X+X A+\left(\begin{array}{c}
0 \\
C
\end{array}\right)^{*} J\left(\begin{array}{c}
0 \\
C
\end{array}\right)-\left[X B+\left(\begin{array}{c}
0 \\
C
\end{array}\right)^{*} J\left(\begin{array}{c}
I \\
D
\end{array}\right)\right] F=0
\end{aligned}
$$

in view of the Riccati equation (39). This concludes the proof of the lemma.

We like to remark that the first part of the above lemma has some interesting consequences in the case that the parameters $A, B, C, D$ in (32) constitute a minimal state-space realization of a continuous-time transfer function $G \in \mathcal{C}$ and the metric $J$ is chosen to be the associated metric $J_{\mathcal{C}}$ (compare Definition 2.1). By comparison with formula (6) it becomes apparent that under the assumptions of (i) $\left(\begin{array}{c}M \\ N\end{array}\right)$ as in (32) then forms a $J_{\mathcal{C}}-\mathrm{RF}$ of $G$; hence Theorem 2.1, in which we establish the existence of these normalized factorizations, 
guarantees that there exists a function $\left(\begin{array}{c}M \\ N\end{array}\right)$ of the form (32) which is as assumed in (i). As has been proved in the lemma, this implies that $W$ is invertible and that the Riccati equation (39) has a stabilizing solution. Now observe that both $W$ and the Riccati equation are completely determined by the realization $(A, B, C, D)$, i.e. independent of the specific $J_{\mathcal{C}}-\mathrm{RF}$ chosen in (32). This means that in the case that $(A, B, C, D)$ is a minimal state-space realization of $G \in \mathcal{C}$, the assumptions of part (ii) concerning the invertibility of $W$ and the existence of a stabilizing solution to the Riccati equation (39) are always met.

The following dual result investigates left factorizations for the continuous-time case; it can be proved completely analogous to Lemma 4.1 using Theorem 3.1,(ii).

Lemma 4.2 (continuous-time case) Let

$$
\left(\begin{array}{ll}
\tilde{M} & \tilde{N}
\end{array}\right)=\left(\begin{array}{c|cc}
A-H C & -H & B-H D \\
\hline \hat{K}_{1} C & \hat{K}_{1} & \hat{K}_{1} D
\end{array}\right)
$$

be a realization of the asymptotically stable function $(\tilde{M} \tilde{N})$, and let $\tilde{M}^{-1}$ be proper rational; moreover, let $J$ be a given metric and define

$$
\bar{W}:=\left[\left(\begin{array}{ll}
I & D
\end{array}\right) J\left(\begin{array}{ll}
I & D
\end{array}\right)^{*}\right] .
$$

(i) Assume that the realization (43) is observable, $\sigma(A-H C) \subseteq \mathbb{C}_{-}$and $\left(\begin{array}{ll}\tilde{M} & \tilde{N}\end{array}\right)$ is $J$-coinner. Then there exists a solution $Y$ to the system of equations

$$
\begin{aligned}
(A-H C) Y+Y(A-H C)^{*}+(-H, B-H D) J(-H, B-H D)^{*} & =0 \\
(-H, B-H D) J\left(\hat{K}_{1}, \hat{K}_{1} D\right)^{*}+Y\left(\hat{K}_{1} C\right)^{*} & =0 \\
\left(\hat{K}_{1}, \hat{K}_{1} D\right) J\left(\hat{K}_{1}, \hat{K}_{1} D\right)^{*} & =I .
\end{aligned}
$$

Furthermore, $\bar{W}$ is invertible, and there holds

$$
\hat{K}_{1}=\bar{D}_{1} \bar{W}^{-\frac{1}{2}},
$$

where $\bar{D}_{1} \bar{D}_{1}^{*}=I$,

$$
H=\left[\left(\begin{array}{ll}
0 & B
\end{array}\right) J\left(\begin{array}{ll}
I & D
\end{array}\right)^{*}+Y C^{*}\right] \bar{W}^{-1}=: \bar{L} \bar{W}^{-1},
$$

and $Y$ is a stabilizing solution of the Riccati equation

$$
\begin{aligned}
& 0=A Y+Y A^{*}+\left(\begin{array}{lll}
0 & B
\end{array}\right) J\left(\begin{array}{ll}
0 & B
\end{array}\right)^{*}-\left[\begin{array}{ll}
Y C^{*}+\left(\begin{array}{ll}
0 & B
\end{array}\right) J\left(\begin{array}{ll}
I & D
\end{array}\right)^{*}
\end{array}\right] \\
& \text { - } \left.\left[\begin{array}{ll}
I & D
\end{array}\right) J\left(\begin{array}{ll}
I & D
\end{array}\right)^{*}\right]^{-1}\left[\left(\begin{array}{ll}
I & D
\end{array}\right) J\left(\begin{array}{ll}
0 & B
\end{array}\right)^{*}+C Y\right] \text {, }
\end{aligned}
$$

i.e. $Y$ solves (50) and

$$
\left.A-\left[Y C^{*}+\left(\begin{array}{ll}
0 & B
\end{array}\right) J\left(\begin{array}{ll}
I & D
\end{array}\right)^{*}\right]\left[\begin{array}{ll}
I & D
\end{array}\right) J\left(\begin{array}{ll}
I & D
\end{array}\right)^{*}\right]^{-1} C
$$

has all eigenvalues in $\mathbb{C}_{-}$. 
(ii) Conversely, assume that $\bar{W}$ is invertible and that there exists a stabilizing solution to the Riccati equation (50); moreover, let the parameters $\hat{K}_{1}$ and $H$ in the realization (43) be given by the formulas (48) respectively (49). Then $\left(\begin{array}{ll}\tilde{M} & \tilde{N}\end{array}\right)$ is J-coinner, and $Y$ solves the system of equations (45)-(47).

The argumentation following Lemma 4.1 also applies to the present situation and shows that for a minimal realization $(A, B, C, D)$ of a continuous-time transfer function $G \in \mathcal{C}$ and $J:=J_{\mathcal{C}}$ the assumptions concerning the invertibility of $\bar{W}$ and the existence of a stabilizing solution to the Riccati equation (50) stated in the second part of the lemma are always satisfied. Moreover, in this case the general Riccati equation (39) reduces to some well-known equations by plugging in for $J$ the different associated metrics. So for $(A, B, C, D) \in A_{n}^{p, m}$ and $J:=J_{S}$ equation (39) is the antistable Riccati equation (cf. Fuhrmann and Ober (1993))

$$
A^{*} X+X A-X B B^{*} X=0,
$$

whereas for $(A, B, C, D) \in L_{n}^{p, m}$ and $J:=J_{L}$ we obtain the generalized control algebraic Riccati equation (cf. Ober and McFarlane (1989))

$$
A^{*} X+X A+C^{*} C-\left(X B+C^{*} D\right)\left(I+D^{*} D\right)^{-1}\left(D^{*} C+B^{*} X\right)=0 .
$$

Next, for $(A, B, C, D) \in B_{n}^{p, m}$, setting $J:=J_{B}$ yields the bounded-real Riccati equation (cf. Opdenacker and Jonckheere (1988))

$$
A^{*} X+X A-C^{*} C-\left(X B-C^{*} D\right)\left(I-D^{*} D\right)^{-1}\left(-D^{*} C+B^{*} X\right)=0 ;
$$

finally, for $(A, B, C, D) \in P_{n}^{m}$ and $J:=J_{P}$ equation (39) is the positive-real Riccati equation (see e.g. Desai and Pal (1984))

$$
A^{*} X+X A-\left(X B+C^{*}\right)\left(D+D^{*}\right)^{-1}\left(C+B^{*} X\right)=0 .
$$

Naturally, we obtain the dual equations of the above Riccati equations by plugging in the different metrics for $J$ into equation (50).

Next, we derive the analogous results to Lemma 4.1 and Lemma 4.2 for the discrete-time case.

Lemma 4.3 (discrete-time case) Let

$$
\left(\begin{array}{c}
M \\
N
\end{array}\right)=\left(\begin{array}{c|c}
A-B F & B K_{1} \\
\hline-F & K_{1} \\
C-D F & D K_{1}
\end{array}\right)
$$

be a realization of the asymptotically stable function $\left(\begin{array}{c}M \\ N\end{array}\right)$, and let $M^{-1}$ be proper rational. Let $J$ be a given metric, and define

$$
W:=\left[\left(\begin{array}{c}
I \\
D
\end{array}\right)^{*} J\left(\begin{array}{c}
I \\
D
\end{array}\right)+B^{*} X B\right] .
$$


(i) Assume that the realization (56) is reachable, $\sigma(A-B F) \subseteq \mathbb{D}$ and $\left(\begin{array}{l}M \\ N\end{array}\right)$ is J-inner. Then there exists a solution $X$ of the system of equations

$$
\begin{aligned}
(A-B F)^{*} X(A-B F)+\left(\begin{array}{c}
-F \\
C-D F
\end{array}\right)^{*} J\left(\begin{array}{c}
-F \\
C-D F
\end{array}\right) & =X, \\
\left(\begin{array}{c}
K_{1} \\
D K_{1}
\end{array}\right)^{*} J\left(\begin{array}{c}
-F \\
C-D F
\end{array}\right)+\left(B K_{1}\right)^{*} X(A-B F) & =0, \\
\left(\begin{array}{c}
K_{1} \\
D K_{1}
\end{array}\right)^{*} J\left(\begin{array}{c}
K_{1} \\
D K_{1}
\end{array}\right)+\left(B K_{1}\right)^{*} X\left(B K_{1}\right) & =I .
\end{aligned}
$$

Furthermore, $W$ is invertible, and there holds

$$
K_{1}=W^{-\frac{1}{2}} D_{1},
$$

where $D_{1}^{*} D_{1}=I$,

$$
F=W^{-1}\left[\left(\begin{array}{l}
I \\
D
\end{array}\right)^{*} J\left(\begin{array}{c}
0 \\
C
\end{array}\right)+B^{*} X A\right]=: W^{-1} L
$$

and $X$ is a stabilizing solution of the Riccati equation

$$
\begin{aligned}
X=A^{*} X A+\left(\begin{array}{c}
0 \\
C
\end{array}\right)^{*} J\left(\begin{array}{c}
0 \\
C
\end{array}\right)-\left[A^{*} X B+\left(\begin{array}{l}
0 \\
C
\end{array}\right)^{*} J\left(\begin{array}{l}
I \\
D
\end{array}\right)\right] \\
\cdot\left[\left(\begin{array}{c}
I \\
D
\end{array}\right)^{*} J\left(\begin{array}{c}
I \\
D
\end{array}\right)+B^{*} X B\right]^{-1}\left[\left(\begin{array}{c}
I \\
D
\end{array}\right)^{*} J\left(\begin{array}{c}
0 \\
C
\end{array}\right)+B^{*} X A\right],
\end{aligned}
$$

i.e. $X$ solves (63) and

$$
A-B\left[\left(\begin{array}{c}
I \\
D
\end{array}\right)^{*} J\left(\begin{array}{l}
I \\
D
\end{array}\right)+B^{*} X B\right]^{-1}\left[\left(\begin{array}{c}
I \\
D
\end{array}\right)^{*} J\left(\begin{array}{c}
0 \\
C
\end{array}\right)+B^{*} X A\right]
$$

has all eigenvalues in $\mathbb{D}$.

(ii) Conversely, assume that $W$ is invertible and that there exists a stabilizing solution $X$ to the Riccati equation (63); moreover, let the parameters $K_{1}$ and $F$ in the realization (56) be given by the formulas (61) respectively (62). Then $\left(\begin{array}{l}M \\ N\end{array}\right)$ is J-inner, and $X$ solves the system of equations (58)-(60).

\section{Proof:}

(i): For the first direction of the proof, assume that $\left(\begin{array}{c}M \\ N\end{array}\right)$ is $J$-inner, $A-B F$ is asymptotically stable and the realization (56) is reachable. Then by Theorem 3.2,(i)(b) there exists a solution $X$ of the system (DIn1)-(DIn3), which in terms of the given realization for $\left(\begin{array}{l}M \\ N\end{array}\right)$ are the equations $(58)-(60)$. Next, we look at equation $(60)$ to observe that

$$
\begin{aligned}
I & =\left(\begin{array}{c}
K_{1} \\
D K_{1}
\end{array}\right)^{*} J\left(\begin{array}{c}
K_{1} \\
D K_{1}
\end{array}\right)+\left(B K_{1}\right)^{*} X\left(B K_{1}\right) \\
& =K_{1}^{*}\left[\left(\begin{array}{c}
I \\
D
\end{array}\right)^{*} J\left(\begin{array}{c}
I \\
D
\end{array}\right)+B^{*} X B\right] K_{1}
\end{aligned}
$$


from this we conclude that $W$ is invertible and $K_{1}$ is of the form (61). Furthermore, equation (59) reads

$$
\begin{aligned}
0 & =\left(\begin{array}{c}
K_{1} \\
D K_{1}
\end{array}\right)^{*} J\left(\begin{array}{c}
-F \\
C-D F
\end{array}\right)+\left(B K_{1}\right)^{*} X(A-B F) \\
& =K_{1}^{*}\left[\left(\begin{array}{c}
I \\
D
\end{array}\right)^{*} J\left(\begin{array}{c}
-F \\
C-D F
\end{array}\right)+B^{*} X(A-B F)\right],
\end{aligned}
$$

and taking into account the invertibility of $K_{1}$ results in

$$
\begin{aligned}
0 & =\left(\begin{array}{l}
I \\
D
\end{array}\right)^{*} J\left(\begin{array}{c}
-F \\
C-D F
\end{array}\right)+B^{*} X(A-B F) \\
& =\left[\left(\begin{array}{c}
I \\
D
\end{array}\right)^{*} J\left(\begin{array}{c}
-I \\
-D
\end{array}\right)-B^{*} X B\right] F+\left(\begin{array}{c}
I \\
D
\end{array}\right)^{*} J\left(\begin{array}{c}
0 \\
C
\end{array}\right)+B^{*} X A
\end{aligned}
$$

respectively

$$
\left[\left(\begin{array}{l}
I \\
D
\end{array}\right)^{*} J\left(\begin{array}{c}
I \\
D
\end{array}\right)+B^{*} X B\right] F=\left(\begin{array}{c}
I \\
D
\end{array}\right)^{*} J\left(\begin{array}{c}
0 \\
C
\end{array}\right)+B^{*} X A .
$$

As has been proved above, $W$ is invertible, and hence we have formula (62) for $F$. In the end, in order to show that $X$ is a stabilizing solution of the Riccati equation (63), we start from (58) to obtain

$$
\begin{aligned}
X= & (A-B F)^{*} X(A-B F)+\left(\begin{array}{c}
-F \\
C-D F
\end{array}\right)^{*} J\left(\begin{array}{c}
-F \\
C-D F
\end{array}\right) \\
= & A^{*} X(A-B F)-F^{*}\left[B^{*} X(A-B F)+\left(\begin{array}{c}
I \\
D
\end{array}\right)^{*} J\left(\begin{array}{c}
-F \\
C-D F
\end{array}\right)\right] \\
& +\left(\begin{array}{c}
0 \\
C
\end{array}\right)^{*} J\left(\begin{array}{c}
-F \\
C-D F
\end{array}\right),
\end{aligned}
$$

which by $(65)$ reduces to

$$
\begin{aligned}
X & =A^{*} X(A-B F)+\left(\begin{array}{l}
0 \\
C
\end{array}\right)^{*} J\left(\begin{array}{c}
-F \\
C-D F
\end{array}\right) \\
& =A^{*} X A+\left(\begin{array}{c}
0 \\
C
\end{array}\right)^{*} J\left(\begin{array}{l}
0 \\
C
\end{array}\right)-\left[A^{*} X B+\left(\begin{array}{l}
0 \\
C
\end{array}\right)^{*} J\left(\begin{array}{c}
I \\
D
\end{array}\right)\right] F .
\end{aligned}
$$

Thus $X$ solves the Riccati equation; recall that we have shown above that $F$ is of the form (62). Finally, it is also clear that $X$ is stabilizing, since by assumption $A-B F$ is asymptotically stable and has the form (64).

(ii): By Theorem 3.2,(i)(a) and the asymptotic stability of $A-B F$ the solvability of the system (58)-(60) is sufficient for $\left(\begin{array}{c}M \\ N\end{array}\right)$ being $J$-inner. Now we assume that $K_{1}$ and $F$ are as given in (61) respectively (62) with $X$ a stabilizing solution of (63); note that $K_{1}$ is necessarily invertible because $M^{-1}$ is proper rational. Looking at equation (61) there holds

$$
\begin{gathered}
\left(\begin{array}{c}
K_{1} \\
D K_{1}
\end{array}\right)^{*} J\left(\begin{array}{c}
K_{1} \\
D K_{1}
\end{array}\right)+\left(B K_{1}\right)^{*} X\left(B K_{1}\right)=K_{1}^{*}\left[\left(\begin{array}{l}
I \\
D
\end{array}\right)^{*} J\left(\begin{array}{l}
I \\
D
\end{array}\right)+B^{*} X B\right] K_{1} \\
=K_{1}^{*} W K_{1}=D_{1}^{*} W^{-\frac{1}{2}} W W^{-\frac{1}{2}} D_{1}=D_{1}^{*} D_{1}=I
\end{gathered}
$$


i.e. (60) holds true. Next, one can reformulate the left-hand side of (59) to obtain

$$
\begin{aligned}
\left(\begin{array}{c}
K_{1} \\
D K_{1}
\end{array}\right)^{*} J\left(\begin{array}{c}
-F \\
C-D F
\end{array}\right)+\left(B K_{1}\right)^{*} X(A-B F) & \\
= & K_{1}^{*}\left[\left(\begin{array}{c}
I \\
D
\end{array}\right)^{*} J\left(\begin{array}{c}
-F \\
C-D F
\end{array}\right)+B^{*} X(A-B F)\right] \\
& =K_{1}^{*}\left[\left[\left(\begin{array}{l}
I \\
D
\end{array}\right)^{*} J\left(\begin{array}{c}
-I \\
-D
\end{array}\right)-B^{*} X B\right] F+\left(\begin{array}{c}
I \\
D
\end{array}\right)^{*} J\left(\begin{array}{l}
0 \\
C
\end{array}\right)+B^{*} X A\right],
\end{aligned}
$$

and thus we get by (61) and (62) that

$$
\begin{aligned}
\left(\begin{array}{c}
K_{1} \\
D K_{1}
\end{array}\right)^{*} J\left(\begin{array}{c}
-F \\
C-D F
\end{array}\right)+\left(B K_{1}\right)^{*} X(A-B F) & =K_{1}^{*}(-W F+L) \\
& =K_{1}^{*}(-W F+W F)=0
\end{aligned}
$$

this means that also (59) is satisfied. In the end, multiply (59) on the left by $\left(K_{1}^{*}\right)^{-1}$ to observe that

$$
\begin{aligned}
(A-B F)^{*} X(A-B F)+\left(\begin{array}{c}
-F \\
C-D F
\end{array}\right)^{*} J\left(\begin{array}{c}
-F \\
C-D F
\end{array}\right) \\
=A^{*} X(A-B F)-F^{*}\left[B^{*} X(A-B F)+\left(\begin{array}{c}
I \\
D
\end{array}\right)^{*} J\left(\begin{array}{c}
-F \\
C-D F
\end{array}\right)\right] \\
\quad+\left(\begin{array}{c}
0 \\
C
\end{array}\right)^{*} J\left(\begin{array}{c}
-F \\
C-D F
\end{array}\right) \\
=A^{*} X(A-B F)+\left(\begin{array}{l}
0 \\
C
\end{array}\right)^{*} J\left(\begin{array}{c}
-F \\
C-D F
\end{array}\right) \\
=A^{*} X A+\left(\begin{array}{l}
0 \\
C
\end{array}\right)^{*} J\left(\begin{array}{l}
0 \\
C
\end{array}\right)-\left[A^{*} X B+\left(\begin{array}{l}
0 \\
C
\end{array}\right)^{*} J\left(\begin{array}{c}
I \\
D
\end{array}\right)\right] F \\
=A^{*} X A+\left(\begin{array}{l}
0 \\
C
\end{array}\right)^{*} J\left(\begin{array}{c}
0 \\
C
\end{array}\right)-L^{*} F \\
=A^{*} X A+\left(\begin{array}{c}
0 \\
C
\end{array}\right)^{*} J\left(\begin{array}{c}
0 \\
C
\end{array}\right)-L^{*} W^{-1} L=X
\end{aligned}
$$

in view of the Riccati equation (63). Hence the proof is completed.

A completely analogous reasoning as in the continuous-time case shows that for a minimal realization $(A, B, C, D)$ of a discrete-time transfer function $G \in \mathcal{C}$ and $J:=J_{\mathcal{C}}$ the assumptions of part (ii) concerning $W$ and the Riccati equation are satisfied. The following dual result is about left factorizations in the discrete-time case.

Lemma 4.4 (discrete-time case) Let

$$
\left(\begin{array}{ll}
\tilde{M} & \tilde{N}
\end{array}\right)=\left(\begin{array}{c|cc}
A-H C & -H & B-H D \\
\hline \hat{K}_{1} C & \hat{K}_{1} & \hat{K}_{1} D
\end{array}\right)
$$

be a realization of the asymptotically stable function $(\tilde{M} \tilde{N})$, and let $\tilde{M}^{-1}$ be proper rational. Let $J$ be a given metric, and define

$$
\bar{W}:=\left[\left(\begin{array}{ll}
I & D
\end{array}\right) J\left(\begin{array}{ll}
I & D
\end{array}\right)^{*}+C Y C^{*}\right] .
$$


(i) Assume that the realization (67) is observable, $\sigma(A-H C) \subseteq \mathbb{D}$ and $\left(\begin{array}{ll}\tilde{M} & \tilde{N}\end{array}\right)$ is $J$-coinner. Then there exists a solution $Y$ of the system of equations

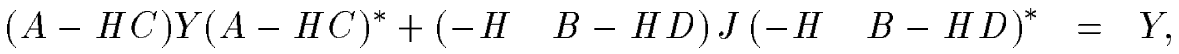

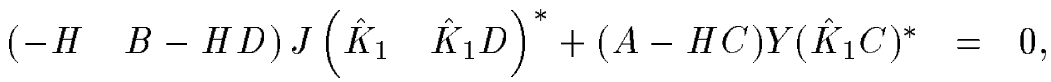

$$
\begin{aligned}
& \left(\begin{array}{ll}
\hat{K}_{1} & \hat{K}_{1} D
\end{array}\right) J\left(\begin{array}{ll}
\hat{K}_{1} & \hat{K}_{1} D
\end{array}\right)^{*}+\left(\hat{K}_{1} C\right) Y\left(\hat{K}_{1} C\right)^{*}=I .
\end{aligned}
$$

Furthermore, $\bar{W}$ is invertible, and there holds

$$
\hat{K}_{1}=\bar{D}_{1} \bar{W}^{-\frac{1}{2}}
$$

where $\bar{D}_{1} \bar{D}_{1}^{*}=I$,

$$
H=\left[\left(\begin{array}{ll}
0 & B
\end{array}\right) J\left(\begin{array}{ll}
I & D
\end{array}\right)^{*}+A Y C^{*}\right] \bar{W}^{-1}=: \bar{L} \bar{W}^{-1},
$$

and $Y$ is a stabilizing solution of the Riccati equation

$$
\begin{aligned}
& Y=A Y A^{*}+\left(\begin{array}{ll}
0 & B
\end{array}\right) J\left(\begin{array}{ll}
0 & B
\end{array}\right)^{*}-\left[A Y C^{*}+\left(\begin{array}{ll}
0 & B
\end{array}\right) J\left(\begin{array}{ll}
I & D
\end{array}\right)^{*}\right] \\
& {\left[\left(\begin{array}{ll}
I & D
\end{array}\right) J\left(\begin{array}{ll}
I & D
\end{array}\right)^{*}+C Y C^{*}\right]^{-1}\left[\left(\begin{array}{ll}
I & D
\end{array}\right) J\left(\begin{array}{ll}
0 & B
\end{array}\right)^{*}+C Y A^{*}\right] \text {, }}
\end{aligned}
$$

i.e. $Y$ solves (74) and

$$
\left.A-\left[A Y C^{*}+\left(\begin{array}{ll}
0 & B
\end{array}\right) J\left(\begin{array}{ll}
I & D
\end{array}\right)^{*}\right]\left[\begin{array}{ll}
I & D
\end{array}\right) J\left(\begin{array}{ll}
I & D
\end{array}\right)^{*}+C Y C^{*}\right]^{-1} C
$$

has all eigenvalues in $\mathbb{D}$.

(ii) Conversely, assume that $\bar{W}$ is invertible and that there exists a stabilizing solution $Y$ to the Riccati equation (74); moreover, let the parameters $\hat{K}_{1}$ and $H$ in the realization (67) be given by the formulas (72) respectively (73). Then $\left(\begin{array}{ll}\tilde{M} & \tilde{N}\end{array}\right)$ is J-coinner, and $Y$ solves the system of equations (69)-(71).

The assumptions in part (ii) are again always fulfilled if we regard a minimal realization $(A, B, C, D)$ of a function $G \in \mathcal{C}$ and the associated metric $J_{\mathcal{C}}$. Furthermore, for these realizations we also in the discrete-time case obtain some equations which are well-known in the control theory literature by plugging in the different associated metrics into the Riccati equation (63) (or the respective dual equations, if we examine Riccati equation (74) instead). So for $(A, B, C, D) \in D A_{n}^{p, m}$ and $J:=J_{S}$ we obtain

$$
X=A^{*} X A-A^{*} X B\left(I+B^{*} X B\right)^{-1} B^{*} X A,
$$

which is the discrete-time antistable Riccati equation. For $(A, B, C, D) \in D L_{n}^{p, m}$ and $J:=J_{L}$, equation $(63)$ reads

$$
X=A^{*} X A+C^{*} C-\left(A^{*} X B+C^{*} D\right)\left(I+D^{*} D+B^{*} X B\right)^{-1}\left(D^{*} C+B^{*} X A\right) ;
$$


this equation is called the discrete-time generalized control algebraic Riccati equation . Next, $(A, B, C, D) \in D B_{n}^{p, m}$ and $J:=J_{B}$ yields the discrete-time bounded-real Riccati equation

$$
X=A^{*} X A-C^{*} C-\left(A^{*} X B-C^{*} D\right)\left(I-D^{*} D+B^{*} X B\right)^{-1}\left(-D^{*} C+B^{*} X A\right) .
$$

Finally, $(A, B, C, D) \in D P_{n}^{m}$ and $J:=J_{P}$ results in the discrete-time positive-real Riccati equation

$$
X=A^{*} X A-\left(A^{*} X B+C^{*}\right)\left(D+D^{*}+B^{*} X B\right)^{-1}\left(C+B^{*} X A\right) .
$$

By now we have finished the technical preparations and finally are in the position to state and prove the main result of this paper; since the procedure is completely analogous in continuous- and discrete-time, we give the theorem for both cases simultaneously and only indicate the modifications required for the discrete-time case in parantheses.

Theorem 4.1 Let $G \in \mathcal{C}$ be a proper rational continuous-time (discrete-time) transfer function, and let $(A, B, C, D)$ be a minimal state-space realization of $G$.

Then all doubly-coprime factorizations $G=N M^{-1}=\tilde{M}^{-1} \tilde{N}$ with the additional properties that $(N, M)$ is a $J_{\mathcal{C}}-R C F,(\tilde{N}, \tilde{M})$ is a $J_{\mathcal{C}}-L C F$ and

$$
\delta\left(\begin{array}{cc}
M & U \\
N & V
\end{array}\right)=n=\delta\left(\begin{array}{cc}
\tilde{V} & -\tilde{U} \\
-\tilde{N} & \tilde{M}
\end{array}\right)
$$

are given by

$$
\begin{aligned}
& \left(\begin{array}{cc}
M & U \\
N & V
\end{array}\right)=\left(\begin{array}{c|cc}
A-B F & B K_{1} & B D_{2}+H \hat{K}_{1}^{-1} \\
\hline-F & K_{1} & D_{2} \\
C-D F & D K_{1} & \hat{K}_{1}^{-1}+D D_{2}
\end{array}\right), \\
& \left(\begin{array}{cc}
\tilde{V} & -\tilde{U} \\
-\tilde{N} & \tilde{M}
\end{array}\right)=\left(\begin{array}{c|cc}
A-H C & H D-B & -H \\
\hline-K_{1}^{-1} F-K_{1}^{-1} D_{2} \hat{K}_{1} C & K_{1}^{-1}+K_{1}^{-1} D_{2} \hat{K}_{1} D & -K_{1}^{-1} D_{2} \hat{K}_{1} \\
\hat{K}_{1} C & -\hat{K}_{1} D & \hat{K}_{1}
\end{array}\right),
\end{aligned}
$$

where $K_{1}$ is of the form (37) ((61)), $\hat{K}_{1}$ is given by formula (48) ((72)), $F$ is as in (38) ((62)), $H$ is given by (49) ((73)) and $X$ and $Y$ are the respective (uniquely determined) stabilizing solutions to the Riccati equations (39) ((63)) and (50) ((74)).

\section{Proof:}

First we prove that every $J_{\mathcal{C}}$-normalized doubly-coprime factorization of $G$ of minimal McMillan degree is of the form given in the statement of the theorem. Hence assume $G=N M^{-1}$ is a $J_{\mathcal{C}}-\mathrm{RF}$. In particular, by Theorem 2.1 there holds $\delta\left(\begin{array}{l}M \\ N\end{array}\right)=n$, which in view of (6) says that

$$
\left(\begin{array}{c}
M \\
N
\end{array}\right)=\left(\begin{array}{c|c}
A-B F & B K_{1} \\
\hline-F & K_{1} \\
C-D F & D K_{1}
\end{array}\right)
$$


with stabilizing $F$ and invertible $K_{1}$. Now the $J_{\mathcal{C}}$-normalization condition means that $\left(\begin{array}{c}M \\ N\end{array}\right)$ is an $J_{\mathcal{C}}$-inner function; moreover, since $\delta\left(\begin{array}{c}M \\ N\end{array}\right)=n$ holds, (82) is a minimal realization. Applying Lemma 4.1,(i) (Lemma 4.3,(i)), we get that $K_{1}$ and $F$ are of the desired form, and that a solution $X$ of the set of equations (34)-(36) ((58)-(60)) exists which also is a stabilizing solution of the Riccati equation (39) ((63)). Observe that by looking at the properties of the Sylvester map (Stein map) we get that $X$ is uniquely determined, since it solves the Lyapunov equation (34) ((58)) and $A-B F$ is asymptotically stable. Summarizing, we have seen so far that every $J_{\mathcal{C}}-\mathrm{RF}$ is of the form (82), and that $K_{1}$ and $F$ are as stated in theorem; moreover, every $J_{\mathcal{C}}-\mathrm{RF}$ gives rise to a stabilizing solution to the Riccati equation (39) ((63)).

The next step is to go through a dual analysis for a $J_{\mathcal{C}}-\mathrm{LF} G=\tilde{M}^{-1} \tilde{N}$. In view of (7) and the fact that by Theorem 2.1 there holds $\delta\left(\begin{array}{ll}-\tilde{N} & \tilde{M}\end{array}\right)=n$, we have a state-space representation

$$
\left(\begin{array}{cc}
-\tilde{N} & \tilde{M}
\end{array}\right)=\left(\begin{array}{c|cc}
A-H C & H D-B & -H \\
\hline \hat{K}_{1} C & -\hat{K}_{1} D & \hat{K}_{1}
\end{array}\right),
$$

where $H$ is a stabilizing output injection and $\hat{K}_{1}$ is invertible; moreover, the statement about the McMillan degree shows that this realization is minimal. The factorization is $J_{\mathcal{C}}$-normalized by assumption, and hence an application of Lemma 4.2,(i) (Lemma 4.4,(i)) proves the formulas for $\hat{K}_{1}$ and $H$ as well as the fact that $Y$, the solution of the system of equations (45)-(47) ((69)-(71)), is a stabilizing solution to the Riccati equation (50) $((74)) . A-H C$ is asymptotically stable, and hence $Y$ is uniquely determined because it solves the Lyapunov equation (45) ((69)). In view of the results obtained so far formulas (80) and (81) follow now directly by plugging in into formulas (8) and (9) for doublycoprime factorizations. This ends the proof of the first implication of the theorem.

For the proof of the backward direction, we assume that we are given the state-space formulas (80) and (81), and that the parameters in these representations are as stated in the theorem. We have to show that the functions given by these formulas constitute a doubly-coprime factorization of $G$ which is $J_{\mathcal{C}}$-normalized and satisfies

$$
\delta\left(\begin{array}{cc}
M & U \\
N & V
\end{array}\right)=n=\delta\left(\begin{array}{cc}
\tilde{V} & -\tilde{U} \\
-\tilde{N} & \tilde{M}
\end{array}\right)
$$

The first observation is that all parameters are well-defined, i.e. $W$ and $\bar{W}$ are indeed invertible and stabilizing solutions to the various Riccati equations exist; this follows in view of the remarks on Lemma 4.1 - Lemma 4.4 by the fact that $(A, B, C, D)$ is a minimal realization of $G$. Moreover, the given formulas constitute a doubly-coprime factorization of $G$ of the desired McMillan degree. This is immediate by comparison with formulas (8) and (9) and the fact that $X$ and $Y$ are the stabilizing solutions to the Riccati equations (39) $((63))$ and (50) $((74))$, which means that $A-B F$ and $A-H C$ are stable. A standard argument from the theory of Riccati equations shows that the stabilizing solution is unique (see e.g. Ionescu and Weiss (1991)). So what remains to be checked is that the $J_{\mathcal{C}^{-}}$ normalization conditions hold. 
Starting from (80), we get the state-space representation

$$
\left(\begin{array}{c}
M \\
N
\end{array}\right)=\left(\begin{array}{c|c}
A-B F & B K_{1} \\
\hline-F & K_{1} \\
C-D F & D K_{1}
\end{array}\right) .
$$

$J_{\mathcal{C}}$-normalization means that $\left(\begin{array}{c}M \\ N\end{array}\right)$ is a $J_{\mathcal{C}}$-inner function, and this property can be proved directly via Lemma 4.1,(ii) (Lemma 4.3,(ii)). On the other hand, (81) gives the state-space representation

$$
\left(\begin{array}{ll}
-\tilde{N} & \tilde{M}
\end{array}\right)=\left(\begin{array}{c|cc}
A-H C & H D-B & -H \\
\hline \hat{K}_{1} C & -\hat{K}_{1} D & \hat{K}_{1}
\end{array}\right),
$$

and the validity of the $J_{\mathcal{C}}$-normalization condition follows by invoking Lemma 4.2 ,(ii) (Lemma 4.4,(ii)) in the same fashion as above. This concludes the proof.

The formulas for the doubly-coprime factorizations are quite complicated and simplify considerably if we regard only particular factorizations; choose e.g. $D_{1}=I=\bar{D}_{1}$ and $D_{2}=0$.

To conclude this paper, we like to mention that several of the results included in the main theorem of the present work have been presented in the literature before. Indeed, the whole case of continuous-time functions has been treated in Fuhrmann and Ober (1993); however, the authors do quite involved state-space calculations separately for each function class. Normalized coprime factorizations for discrete-time antistable and minimal functions have been examined in Meyer (1990) and Chu (1988). In both these references a proof is given only for the special case of strictly proper transfer functions, and it is additionally assumed that the denominator matrices $M$ and $\tilde{M}$ in the factorization have no pole at the origin. Bongers and Heuberger (1990) use the state-space characterization for inner functions as contained in Theorem $3.2\left(J_{\mathcal{C}}=I\right)$ to obtain formulas for the factorization of transfer functions in $D L_{n}^{p, m}$.

We also want to emphasize that we have implicitely proved existence of stabilizing solutions for the Riccati equations (52)-(55) and (76)-(79) (and the dual equations); as has been remarked in the text, these results are obtained from the existence of normalized factorizations, which has been established in Theorem 2.1 by exclusively using polynomial methods. Moreover, in the case of bounded real and positive real functions either in continuous- or discrete-time, this leads to alternative proofs for the Bounded-Real Lemma and the Positive-Real Lemma (cf. e.g. Anderson and Vongpanitlerd (1973)); these results have not been included here because of reasons of length and can be found in Hoffmann (1994).

\section{Conclusions}

The aim of this paper was to derive state-space formulas for normalized doubly-coprime factorizations for different classes of continuous- and discrete-time transfer functions, namely for antistable, minimal, bounded real and positive real functions; the normalization conditions are adapted for the respective system classes depending on the special structure the examined functions display. 
Our method is based on the observation that normalization means $J_{\mathcal{C}}$-innerness of the matrices containing the factors of the factorization; state-space characterizations of this property are derived in the continuous- as well as in the discrete-time case. Thus, by taking the well-known state-space formulas for general coprime factorizations over $R H_{+}^{\infty}$ and plugging them in into the algebraic characterization of $J$-inner functions, we obtain a parametrization of all normalized doubly-coprime factorizations of a given transfer function in terms of the stabilizing solutions of general degenerate continuous- respectively discrete-time Riccati equations.

We finally like to mention that some of the results presented in this paper have appeared in the control literature before. However, we think that the present work constitutes an interesting contribution to the literature, since our emphasis is not on doing repeated calculations for the different function classes and time axes as in the previous work; instead, we describe a very natural approach to the problem by presenting a unified construction method which contains all earlier results (and several new ones) as special cases.

\section{References}

B. D. O. Anderson and S. Vongpanitlerd, Network Analysis and Synthesis, Prentice-Hall, Englewood Cliffs, New Jersey, (1973).

H. Bart, I. Gohberg and M. A. Kaashoek, Minimal Factorization of Matrix and Operator Functions, Birkhäuser, Basel, (1979).

J. Bognar, Indefinite Inner Product Spaces, Springer Verlag, New York, (1974).

P. M. M. Bongers and P. S. C. Heuberger, Discrete normalized coprime factorization, Proc. 9th Int. Conf., Antibes, France, in: Lect. Notes Control Inf. Sci., 144:307-313, (1990).

C. C. Chu, On discrete inner-outer and spectral factorizations, Proc. Amer. Control Conf., Atlanta, Georgia, 1699-1700, (1988).

U. B. Desai and D. Pal, A transformation approach to stochastic model reduction, IEEE Trans. Automat. Control, 29:1097-1100, (1984).

B. Francis, $A$ course in $H_{\infty}$ Control Theory, Springer Verlag, New York, (1987).

P.A. Fuhrmann, A duality theory for robust control and model reduction, Lin. Alg. Appl., 203-204:471-578, (1994).

P. A. Fuhrmann and J. Hoffmann, Factorization theory for stable, discrete-time inner functions, to appear in Journ. of Mathematical Systems, Estimation and Control, (1994).

P. A. Fuhrmann and R. J. Ober, State space formulas for coprime factorizations, the T. Ando Anniversary Volume, in: Operator Theory: Advances and Applications, Birkhäuser, Basel, 62:39-75, (1993).

K. Glover, All optimal Hankel-norm approximations and their $L^{\infty}$-error bounds, Int. J. Contr., 39(6):1115-1193, (1984). 
J. Hoffmann, Balancing and Factorization Theory for Classes of Linear Systems in Discreteand Continuous-Time, Doctoral Dissertation, University of Kaiserslautern, Department of Mathematics, (1994).

K. Hoffmann, Banach spaces of analytic functions, Prentice-Hall, Englewoods Cliffs, NJ, (1962).

V. Ionescu and M. Weiss, On computing the stabilizing solution of the discrete-time Riccati equation, Lin. Alg. Appl., 174:229-238, (1991).

P. Khargonekar and E. Sontag, On the relation between stable matrix factorizations and regulable realizations of linear systems over rings, IEEE Trans. Automat. Control, 27:627$638,(1982)$.

V. Kucera, Discrete Linear Control, John Wiley \& Sons, New York, (1979).

D. McFarlane and K. Glover, Robust Controller Design using Normalized Coprime Factor Plant Descriptions, Lecture Notes in Control and Information Sciences, vol. 10, Springer, New York, (1989).

D. G. Meyer, The construction of special coprime factorizations in discrete time, IEEE Trans. Autom. Control, 35(5):588-590, (1990).

C. N. Nett, C. A. Jacobson and M. J. Balas, A connection between state-space and doubly coprime fractional representations, IEEE Trans. Autom. Control, 29:831-832, (1984).

R. J. Ober and D. C. McFarlane, Balanced canonical forms for minimal systems: a normalized coprime factor approach, Lin. Alg. Appl., 122-124:23-64, (1989).

P. C. Opdenacker and E. A. Jonckheere, A contraction mapping preserving balanced reduction scheme and its infinity norm error bound, IEEE Trans. Circ. and Sys., 35:184-189, (1988).

M. Vidyasagar, Control System Synthesis: A Coprime Factorization Approach, M.I.T. Press, Cambridge, MA, (1985).

W. M. Wonham, Linear Multivariable Control: A Geometric Approach, Springer, New York, (1979).

D. C. Youla, J. J. Bongiorno and H. A. Jabr, Modern Wiener-Hopf design of optimal controllers, Part 2: The multivariable case, IEEE Trans. Autom. Control, 21:319-338, (1976). 